\title{
GMR
}

\section{Phenotypic classification of gastric signet ring cell carcinoma and its relationship with K-ras mutation}

\author{
Z.F. Xiong ${ }^{1}$, J. Shi ${ }^{2}$, Z.H. Fu ${ }^{3}$, H.P. Wan ${ }^{1}$ and L.X. Tu${ }^{1}$ \\ ${ }^{1}$ Department of Pathology, \\ First Affiliated Hospital of Nanchang University, Nanchang, China \\ ${ }^{2}$ Department of General Surgery, \\ First Affiliated Hospital of Nanchang University, Nanchang, China \\ ${ }^{3}$ Department of Burns, \\ First Affiliated Hospital of Nanchang University, Nanchang, China \\ Corresponding author: J. Shi \\ E-mail: shiyuan_2016a@163.com
}

Genet. Mol. Res. 16 (2): gmr16029181

Received September 6, 2016

Accepted March 8, 2017

Published April 28, 2017

DOI http://dx.doi.org/10.4238/gmr16029181

Copyright $(2017$ The Authors. This is an open-access article distributed under the terms of the Creative Commons Attribution ShareAlike (CC BY-SA) 4.0 License.

\begin{abstract}
We aimed to analyze gastric signet ring cell (SRC) carcinoma subtypes by investigating gastric and intestinal phenotypic marker expression, and explore the relationship between phenotype and K-ras mutation. Immunohistochemistry was performed on 163 SRC carcinoma patient specimens to detect gastric (MUC1, MUC5AC, and MUC6) and intestinal (MUC2 and CDX2) phenotypic markers, and tumors were classified into gastric $(\mathrm{G})$, intestinal (I), and gastrointestinal (GI) phenotypes. DNA was extracted from the formalin-fixed, paraffinembedded tumor samples, and K-ras mutations in codons 12, 13, and 61 were identified using polymerase chain reaction-based direct DNA sequencing. G, GI, and I phenotypes were observed in 63 (38.6\%), 71 (43.5\%), and 29 cases (17.8\%), respectively. Expression of MUC2 was significantly associated with invasion depth and lymph node
\end{abstract}

Genetics and Molecular Research 16 (2): gmr16029181 
metastasis ( $\mathrm{P}=0.001$ and 0.002 , respectively), whereas that of CDX2 significantly corresponded to tumor size and submucosal invasion ( $\mathrm{P}$ $=0.004$ and 0.001 , respectively). MUC5AC expression was inversely associated with gastric wall invasion $(\mathrm{P}=0.001)$. Intestinal phenotypic marker expression was positively associated with gastric wall invasion and lymph node metastasis. K-ras mutations, all of which were in codon 12 , were detected in $20(12.27 \%)$ tumors, were significantly associated with the I phenotype, and exhibited an inverse relationship with MUC5AC and MUC6 expression. I-phenotype SRC carcinomas should be distinguished from those of the $G$ phenotype because of their increased malignancy regarding invasion and metastasis, and higher K-ras aberration rate. The different K-ras mutation frequencies observed imply distinct genetic mechanisms in the carcinogenesis of I- and G-phenotype gastric SRC carcinomas.

Key words: Gastric carcinoma; Signet ring cell; Phenotype; Mutation; Immunohistochemistry

\section{INTRODUCTION}

Gastric adenocarcinoma is the fourth most common malignancy and the second leading cause of cancer-related mortality worldwide (Humar et al., 2007). It has been reported that $3.4-29 \%$ of patients with gastric adenocarcinoma demonstrate signet ring cell (SRC) histology (Senapati et al., 2008). Compared with other types of gastric adenocarcinoma, SRC carcinoma tends to be more common among young and female individuals (Yokota et al., 1998). It is characterized by its high frequency of lymph node metastasis, infiltrative growth to the gastric wall, and poor prognosis (Otsuji et al., 1998).

Lauren (1965) classified gastric adenocarcinoma into two major histological types, intestinal and diffuse, based on gland formation tendency. According to this system, gastric SRC carcinoma belongs to the diffuse category. With respect to the histogenesis of these two gastric adenocarcinoma types, intestinal malignancies are thought to result from a multistage process beginning with intestinal metaplasia followed by dysplasia and ultimately, gastric cancer. In contrast, cancers of the diffuse type are believed to arise from the normal gastric mucosa, with no precursor lesion (Tajima et al., 2006). It is believed that these two tumor types involve different genetic pathways during carcinogenesis (Tahara et al., 1996). However, recent reports have shown that both gastric and intestinal phenotypic markers are expressed in gastric adenocarcinoma in a manner that imitates the tissue of origin, irrespective of histological type (Tajima et al., 2001). Tumor phenotypic marker expression has also been associated with tumor aggressiveness in this disease. Tajima et al. $(2001,2004)$ reported that among patients with gastric carcinomas of the intestinal type, those with gastric $(\mathrm{G})$-phenotype tumors are at significantly higher risk of peritoneal recurrence and poorer outcome compared to those with intestinal (I)-phenotype malignancies. Conversely, it has been reported that a considerable proportion of gastric SRC carcinomas express intestinal phenotypic markers such as CDX2 and MUC2, and that in this disease, the I phenotype is associated with larger tumors and deeper gastric wall invasion (Bamba et al., 2001; Aihara et al., 2004). Therefore, phenotypic marker expression is closely related to both gastric tumorigenesis and the behavior of gastric adenocarcinoma. It can thus be conjectured that biological differences

Genetics and Molecular Research 16 (2): gmr16029181 
exist between SRC carcinomas of the G and I phenotypes.

Members of the ras family are likely the most important proto-oncogenes in human tumorigenesis. It has been reported that up to $28 \%$ of patients with gastric adenocarcinoma carry K-ras mutations (Lee et al., 1995; Arber et al., 1997). Hongyo et al. (1995) reported that carcinoma of the intestinal type often closely resembles colorectal cancer, in that activation of K-ras by point mutation occurs relatively early in tumor progression. Correspondingly, $\mathrm{K}$-ras mutation has been found to be frequent in intestinal-type cases in Japan (Hongyo et al., 1995). However, only limited data are available regarding the frequency of K-ras mutation in diffuse-type cancers such as SRC carcinoma. Moreover, the relationship between expression of phenotypic markers and genetic alterations is unknown. To the best of our knowledge, no studies of phenotypic marker expression and K-ras mutation in gastric SRC carcinoma involving a large number of cases have been carried out. The association between phenotypic marker profile and K-ras mutation in this disease remains unclear.

In the present study, the expression of phenotypic markers and presence of K-ras gene mutations were examined in 163 cases of gastric SRC carcinoma. The purpose of this work was to clarify the expression of various phenotypic markers in this condition and its relationship with K-ras aberration.

\section{MATERIAL AND METHODS}

\section{Tissues}

In total, 163 patients with gastric SRC carcinoma having undergone gastrectomy between January, 2008 and December, 2013 at the First Affiliated Hospital of Nanchang University (Jiangxi, China) were included. SRC carcinoma was defined as an adenocarcinoma predominantly $(>50 \%)$ comprising isolated or small groups of malignant cells with eccentric crescent-shaped nuclei pushed by intracytoplasmic mucus against the cell membranes. The study group included 95 women and 68 men, with a median age of 51 years (range: 21-82 years). Gastric carcinoma stage was determined according to the International Union Against Cancer 1997 tumor-node-metastasis (TNM) classification of malignant tumors. Written informed consent was obtained from all participants to perform the present work, and the study was approved by the Nanchang University Ethics Committee.

\section{Phenotypic classification of SRC carcinoma}

To classify gastric SRC carcinoma phenotypes, we used MUC2 and CDX2 as intestinal markers. MUC2 is a specific marker of goblet cells, whereas CDX2 is expressed in intestinal epithelial cells and ectopically expressed in intestinal metaplasia and gastric carcinomas of the intestinal type (Jung et al., 2007). We chose MUC1, MUC5AC, and MUC6 as markers of the G phenotype, as these are commonly used for this purpose. MUC1 and MUC5AC are expressed in the superficial foveolar epithelium, whereas MUC6 is expressed in the mucous neck cells of the gastric body and deeper glands of the antrum (Guillem et al., 2000).

According to marker expression, tumors were classified into three different phenotypes, namely, $G$ (positive staining for one or more G-phenotype markers, but no I-phenotype markers), I (positive staining for one or more I-phenotype markers, but no G-phenotype markers), and gastrointestinal (GI; positive staining for both G- and I-phenotype markers).

Genetics and Molecular Research 16 (2): gmr16029181 


\section{Immunohistochemistry}

Tissue specimens were fixed in 10\% formalin and embedded in paraffin. One paraffinembedded tumorous tissue sample was selected for each case and cut into 4- $\mu \mathrm{m}$ sections. The sections were placed in an oven at $60^{\circ} \mathrm{C}$ for $4 \mathrm{~h}$, deparaffinized in xylene, rehydrated in a graded ethanol series, and treated with 3\% hydrogen peroxide solution for $20 \mathrm{~min}$. Antigen retrieval was conducted by heating samples at over $90^{\circ} \mathrm{C}$ for $20 \mathrm{~min}$ in $0.01 \mathrm{M}$ sodium citrate buffer, $\mathrm{pH}$ 6.0, using a microwave. The sections were then incubated with primary antibodies at appropriate dilutions (Table 1 ) at $4^{\circ} \mathrm{C}$ overnight. Bound primary antibodies were detected using Powervision two-step histostaining reagent (PV-6001; Dako, Glostrup, Denmark) as the secondary antibody. Finally, the slides were stained with 3,3'-diaminobenzidine and counterstained with hematoxylin. Samples of normal gastric and normal small intestinal mucosa were used as positive controls for G- and I-phenotype markers, respectively. Negative controls were created by replacing the primary antibodies with phosphate buffered saline.

Table 1. Antibodies used for immunohistochemistry.

\begin{tabular}{l|c|c|l|l}
\hline Antibody & Clone & Dilution & Specificity & Company \\
\hline G-phenotype markers & & & & \\
\hline MUC1 & 45M1 & $1: 100$ & Peptide core of MUC1 & DAKO (Carpinteria, CA, USA) \\
\hline MUC5AC & CLH2 & $1: 100$ & MUC5AC glycoprotein & DAKO \\
\hline MUC6 & CLH5 & $1: 50$ & MUC6 glycoprotein & DAKO \\
\hline I-phenotype markers & & & & \\
\hline MUC2 & Ccp58 & $1: 100$ & MUC2 glycoprotein & DAKO \\
\hline CDX2 & CDX2-88 & $1: 50$ & Peptide core of CDX2 & DAKO \\
\hline
\end{tabular}

Two experienced pathologists blind to the clinicopathologic data independently examined the staining results. At least 10 high-power fields were chosen randomly at $400 \mathrm{X}$ magnification, and $>1000$ tumor cells were counted in each section. Expression of MUC1, MUC2, MUC5AC, and MUC6 was observed in the cytoplasm and cell membrane, and that of CDX2 was localized to the nucleus. Cases were defined as positive when the number of positive tumor cells was $>10 \%$ in each section $(6,15,16)$.

\section{Analysis of K-ras mutation}

\section{DNA extraction}

Microdissection and DNA extraction were performed on 8-10 paraffin-embedded tissue sections, each $8 \mu \mathrm{m}$ in thickness. Using the hematoxylin-eosin(HE)-stained section as a guide, precisely identified tumor tissue was obtained with care using a needle, to ensure that more than $75 \%$ of the recovered material constituted tumor cells, rather than unremarkable connective tissue components, necrotic debris, or cell populations associated with inflammation or hemorrhage. The procured tissue was suspended in $1000 \mu \mathrm{L}$ lysis buffer [50 mM Tris, $1 \mathrm{mM}$ ethylenediaminetetraacetic acid (EDTA), 2\% sodium dodecyl sulfate, and $20 \mu \mathrm{g} / \mathrm{mL}$ proteinase $\mathrm{K}]$ and incubated at $55^{\circ} \mathrm{C}$ overnight. Following its extraction using the phenol-chloroform method, genomic DNA was washed with $75 \%$ ethanol and dissolved in 40 $\mu \mathrm{L}$ Tris-EDTA buffer for use in polymerase chain reaction (PCR). 


\section{PCR assay}

PCR was carried out in a PTC-200 Peltier Thermal Cycler (Bio-Rad, Hercules, CA, USA). A 227-bp sequence across codons 12 and 13, and a 213-bp sequence in codon 61 of the K-ras gene were amplified using the following primer pairs, respectively: 5'-AGG-CCT-GCTGAA-AAT-GAC-TG-3' (sense) and 5'-TCA-AAG-AAT-GGT-CCT-GCA-CC-3' (antisense); and 5'-TGT-AAT-AAT-CCA-GAC-TGT-GTT-TCT-CC-3' (sense) and 5'-AGC-TTA-TTATAT-TCA-ATT-TAA-ACC-CAC-C-3' (antisense). The PCR mixture $(50 \mu \mathrm{L})$ contained 2 $\mu \mathrm{L}$ genomic DNA, $25 \mu \mathrm{L}$ SYBR Green Realtime PCR Master Mix (TOYOBO Co., Ltd., Osaka, Japan), $19 \mu \mathrm{L}$ double-distilled $\mathrm{H}_{2} \mathrm{O}$, and $2 \mu \mathrm{L}$ each primer. Template DNA was initially denatured for $7 \mathrm{~min}$ at $94^{\circ} \mathrm{C}$, then 35 cycles of amplification were carried out, each comprising $45 \mathrm{~s}$ at $94^{\circ} \mathrm{C}, 45 \mathrm{~s}$ at $62^{\circ} \mathrm{C}$, and $45 \mathrm{~s}$ at $72^{\circ} \mathrm{C}$. The reaction was incubated at $72^{\circ} \mathrm{C}$ for $7 \mathrm{~min}$ after the final cycle, and cooled to $4^{\circ} \mathrm{C}$ before DNA sequencing. Negative controls lacking a DNA template were also processed to exclude the possibility of reagent contamination. Samples from colorectal carcinomas known to be homozygous for K-ras mutations in codons 12, 13, and 61 were used as positive controls.

\section{DNA sequencing}

PCR products from each sample were purified with a QIAquick PCR Purification Kit (QIAGEN, Hilden, Germany) and sequenced on an ABI 3730xl sequencer (Genomics Company, Shanghai, China) using a BigDye Terminator v2.0 kit following the manufacturer protocol. Both strands were sequenced for each product, and genomic DNA from control samples was sequenced in parallel to confirm mutations.

\section{Statistical analysis}

All data were analyzed using the SPSS 15.0 software (SPSS Inc., Chicago, IL, USA). Associations between protein expression, K-ras mutation, and various clinicopathologic parameters were analyzed using the chi-square test, two-sided Fisher's test, and Spearman's rank correlation analysis. P values $\leq 0.05$ were considered to represent statistical significance.

\section{RESULTS}

\section{Expression of phenotypic markers and classification of gastric SRC carcinoma phenotype}

Both gastric and intestinal markers showed a heterogeneous staining pattern (Figure 1). Expression of MUC1, MUC5AC, MUC6, MUC2, and CDX2 was observed in 20.9 (34), $73.6(120), 28.8$ (47), 46.6 (76), and 39.3\% (64) of the 163 gastric SRC carcinomas, respectively.

According to the expression of phenotypic markers, the 163 cases were classified into three phenotypes: 63 (38.6\%) exhibited the G phenotype, 71 (43.5\%) the GI phenotype, and $29(17.9 \%)$ the I phenotype. 

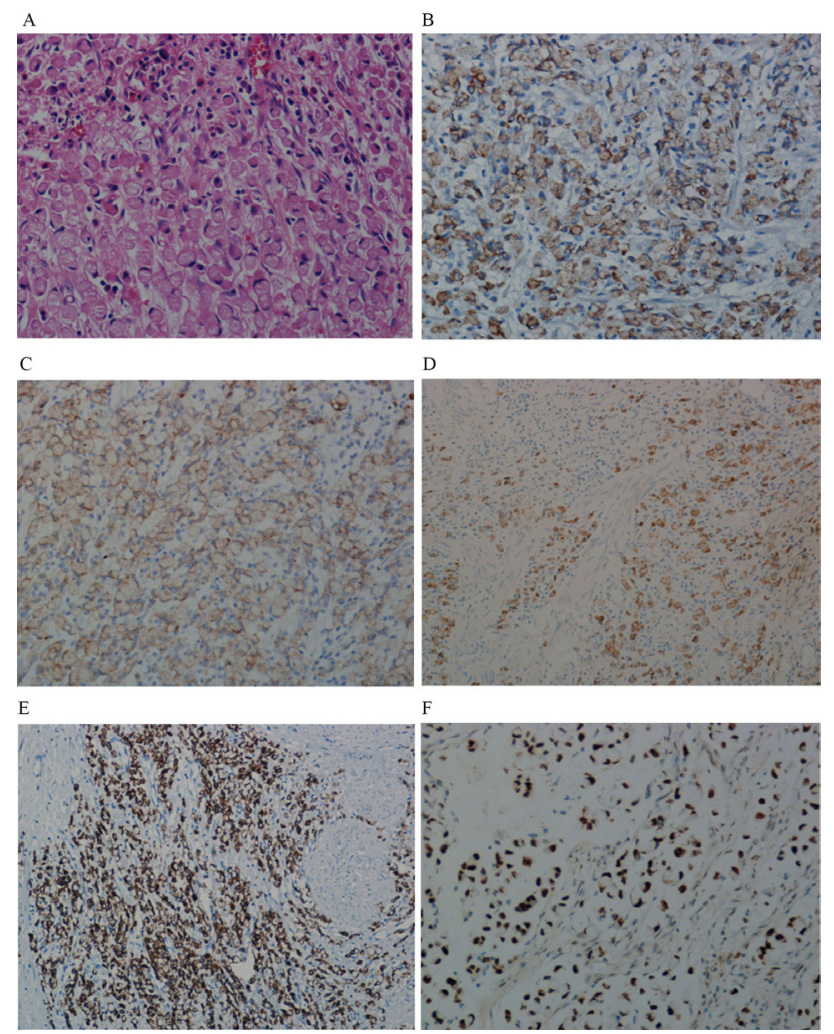

Figure 1. Signet ring cell adenocarcinoma of the gastrointestinal phenotype showing positive MUC1, MUC5AC, MUC6, MUC2, and CDX2 immunohistochemical staining. A. Hematoxylin and eosin (400X magnification); B. MUC1 [3,3'-diaminobenzidine (DAB), 400X]; C. MUC5AC (DAB, 400X); D. MUC6 (DAB, 400X); E. MUC2 (DAB, 200X); F. CDX2 (DAB, 200X). Positive staining for MUC1, MUC5AC, MUC6, and MUC2 can be seen in the signet ring cell cytoplasm and/or membrane, and that for $\mathrm{CDX} 2$ in the nucleus.

\section{Relationship between phenotypic markers and clinicopathologic parameters}

The relationship between phenotypic marker expression and clinicopathologic parameters is shown in Table 2. Compared with MUC2-negative tumors, MUC2-positive malignancies demonstrated a significantly higher lymph node metastasis rate (31.0 vs 55.3\%, respectively, $\mathrm{P}=0.002)$ and deeper wall invasion (51.7 vs $80.3 \%$, respectively, $\mathrm{P}=0.001$ ). No significant correlation was found between MUC2 expression and sex, age, vascular invasion, neural invasion, or TNM stage. In comparison to those negative for CDX2, CDX2positive tumors were significantly larger (diameter $>5.0 \mathrm{~cm} ; 48.5 v s 53.2 \%$, respectively, $\mathrm{P}=$ 0.004 ) and were associated with higher TNM stage (III+IV; 39.4 s $46.9 \%$, respectively, $\mathrm{P}=$ 0.002 ) and deeper wall invasion (47.5 vs 92.2\%, respectively, $\mathrm{P}=0.001)$. CDX2 expression showed no relationship with other clinicopathologic parameters. Positive MUC5AC staining was inversely associated with submucosal invasion [56.7 (positive) $v s 88.4 \%$ (negative), $\mathrm{P}=$ 0.001]. However, no significant correlation was established between MUC5AC expression and other clinicopathologic characteristics. No significant associations were observed between expression of MUC1 or MUC6 and any of the parameters examined. 
Table 2. Associations between phenotypic marker expression $[\mathrm{N}(\%)]$ and clinicopathologic features.

\begin{tabular}{|c|c|c|c|c|c|c|c|c|c|c|c|}
\hline \multirow[t]{2}{*}{ Factor } & \multirow[t]{2}{*}{ Cases } & \multicolumn{2}{|c|}{ MUC2 expression } & \multicolumn{2}{|c|}{ CDX2 expression } & \multicolumn{2}{|c|}{ MUC1 expression } & \multicolumn{2}{|c|}{ MUC5AC expression } & \multicolumn{2}{|c|}{ MUC6 expression } \\
\hline & & $\begin{array}{l}\text { Positive } \\
(\mathrm{N}=76) \\
\end{array}$ & $\begin{array}{l}\text { Negative } \\
(\mathrm{N}=87)\end{array}$ & $\begin{array}{l}\text { Positive } \\
(\mathrm{N}=64) \\
\end{array}$ & $\begin{array}{l}\text { Negative } \\
(\mathrm{N}=99)\end{array}$ & $\begin{array}{l}\text { Positive } \\
(\mathrm{N}=34)\end{array}$ & $\begin{array}{l}\text { Negative } \\
(\mathrm{N}=129)\end{array}$ & $\begin{array}{c}\text { Positive } \\
(\mathrm{N}=120)\end{array}$ & $\begin{array}{l}\text { Negative } \\
(\mathrm{N}=43)\end{array}$ & $\begin{array}{l}\text { Positive } \\
(\mathrm{N}=47)\end{array}$ & $\begin{array}{r}\text { Negative } \\
(\mathrm{N}=116)\end{array}$ \\
\hline \multicolumn{12}{|l|}{$\begin{array}{l}\text { Gender } \\
\end{array}$} \\
\hline Female & 95 & $46(60.5)$ & $49(56.3)$ & $40(62.5)$ & $55(55.6)$ & $18(52.9)$ & $77(59.7)$ & $73(60.8)$ & $22(51.2)$ & $31(66.0)$ & $64(55.2)$ \\
\hline Male & 68 & $30(39.5)$ & $38(43.7)$ & $24(37.5)$ & $44(44.4)$ & $16(47.1)$ & $52(40.3)$ & $47(39.2)$ & $21(48.8)$ & $16(34.0)$ & $52(44.8)$ \\
\hline \multicolumn{12}{|c|}{ Age (years) } \\
\hline$\leq 50$ & 62 & $32(42.1)$ & $30(34.5)$ & $27(42.2)$ & $35(35.4)$ & $17(50.0)$ & $45(34.9)$ & $46(38.3)$ & $16(37.2)$ & $15(31.9)$ & $47(40.5)$ \\
\hline$>50$ & 101 & $44(57.9)$ & $57(65.5)$ & $37(57.8)$ & $64(64.6)$ & $17(50.0)$ & $84(65.1)$ & $74(61.7)$ & $27(62.8)$ & $32(68.1)$ & $69(59.5)$ \\
\hline \multicolumn{12}{|c|}{ Tumor diameter } \\
\hline$\leq 5.0 \mathrm{~cm}$ & 81 & $35(46.1)$ & $46(52.9)$ & $30(46.8)$ & $51(51.5)$ & $13(38.2)$ & $68(52.7)$ & $61(50.8)$ & $20(46.5)$ & $23(48.9)$ & $58(50.0)$ \\
\hline$>5.0 \mathrm{~cm}$ & 82 & $41(53.9)$ & $41(47.1)$ & $34(53.2)^{\mathrm{a}}$ & $48(48.5)^{\mathrm{a}}$ & $21(61.8)$ & $61(47.3)$ & $59(49.2)$ & $23(53.5)$ & $24(51.1)$ & $58(50.0)$ \\
\hline \multicolumn{12}{|c|}{ Depth of wall invasion } \\
\hline $\mathrm{T} 1$ & 57 & $15(19.7)^{b}$ & $42(48.3)^{\mathrm{b}}$ & $5(7.8)^{\mathrm{c}}$ & $52(52.5)^{\mathrm{c}}$ & $8(23.5)$ & $49(38.0)$ & $52(43.3)^{\mathrm{d}}$ & $5(11.6)^{d}$ & $11(23.4)$ & $46(39.7)$ \\
\hline $\mathrm{T} 2$ & 34 & $22(28.9)$ & $12(13.8)$ & $25(39.1)$ & $9(9.1)$ & $5(14.7)$ & $29(22.5)$ & $18(15.0)$ & $16(37.2)$ & $14(29.8)$ & $20(17.2)$ \\
\hline $\mathrm{T} 3$ & 54 & $29(38.2)$ & $25(28.7)$ & $28(43.8)$ & $26(26.3)$ & $17(50.0)$ & $37(28.7)$ & $37(30.8)$ & $17(39.5)$ & $19(40.4)$ & $35(30.2)$ \\
\hline $\mathrm{T} 4$ & 18 & $10(13.2)$ & $8(9.2)$ & $6(9.4)$ & $12(12.1)$ & $4(11.8)$ & $14(10.8)$ & $13(10.8)$ & $5(11.6)$ & $3(6.4)$ & $15(12.9)$ \\
\hline \multicolumn{12}{|c|}{ Vascular invasion } \\
\hline Positive & 32 & $18(23.7)$ & $14(16.1)$ & $15(23.4)$ & $17(17.2)$ & $7(20.6)$ & $25(19.4)$ & $23(19.2)$ & $9(20.9)$ & $12(25.5)$ & $20(17.2)$ \\
\hline Negative & 131 & $58(76.3)$ & $73(83.9)$ & $49(76.6)$ & $82(82.8)$ & $27(79.4)$ & $104(80.6)$ & $97(80.8)$ & $34(79.1)$ & $35(74.5)$ & $96(82.8)$ \\
\hline \multicolumn{12}{|c|}{ Neural invasion } \\
\hline Positive & 52 & $27(35.5)$ & $25(28.7)$ & $26(40.6)$ & $26(26.2)$ & $10(29.4)$ & $42(32.6)$ & $37(30.8)$ & $15(34.8)$ & $17(36.2)$ & $35(30.2)$ \\
\hline Negative & 111 & $49(64.5)$ & $62(71.3)$ & $38(59.4)$ & $73(73.8)$ & $24(70.6)$ & $87(67.4)$ & $83(69.2)$ & $28(65.2)$ & $30(63.8)$ & $81(69.8)$ \\
\hline \multicolumn{12}{|c|}{ Lymph node metastasis } \\
\hline Positive & 69 & $42(55.3)^{\mathrm{c}}$ & $27(31.0)^{\mathrm{e}}$ & $31(48.4)$ & $38(38.4)$ & $19(55.9)$ & $50(38.8)$ & $46(38.3)$ & $23(53.5)$ & $24(51.1)$ & $45(38.8)$ \\
\hline Negative & 94 & $34(44.7)$ & $60(69.0)$ & $33(51.6)$ & $61(61.6)$ & $15(44.1)$ & $79(61.2)$ & $74(61.7)$ & $20(46.5)$ & $23(48.9)$ & $71(61.2)$ \\
\hline \multicolumn{12}{|c|}{$\begin{array}{l}\text { TNM stage } \\
\end{array}$} \\
\hline I+II & 94 & $43(56.6)$ & $51(58.6)$ & $34(53.1)$ & $60(60.6)$ & $17(50.0)$ & $77(59.7)$ & $77(64.2)$ & $17(39.5)$ & $27(57.4)$ & $67(57.7)$ \\
\hline III + IV & 69 & $33(43.4)$ & $36(41.4)$ & $30(46.9)^{f}$ & $39(39.4)^{f}$ & $17(50.0)$ & $52(40.3)$ & $43(35.8)$ & $26(60.5)$ & $20(42.6)$ & $49(42.3)$ \\
\hline
\end{tabular}

$\mathrm{TNM}=$ tumor-node-metastasis. ${ }^{\mathrm{a}} \mathrm{P}=0.004$ (CDX2 expression); ${ }^{\mathrm{b}} \mathrm{P}=0.001$ (MUC2 expression); ${ }^{\mathrm{c}} \mathrm{P}=0.001(\mathrm{CDX} 2$ expression); ${ }^{\mathrm{d}} \mathrm{P}=0.001$ (MUC5AC expression); ${ }^{\mathrm{e}} \mathrm{P}=0.002$ (MUC2 expression); ${ }^{\mathrm{P}}=0.002$ (CDX2 expression). b,c,d $\mathrm{T} 1$ vs $\mathrm{T} 2+\mathrm{T} 3+\mathrm{T} 4$.

\section{Relationship between phenotypic classification and clinicopathologic parameters}

Associations between tumor phenotypes and clinicopathologic parameters are shown in Table 3. The proportion of patients with lymph node metastasis was $34.9(22 / 63), 40.8$ (29/71), and 62.1\% (18/29), in the G-, GI-, and I-phenotype groups, respectively. The G phenotype was associated with a significantly lower rate of lymph node metastasis than the other phenotypes identified $(\mathrm{P}<0.01)$. Compared to those with tumors of the $\mathrm{G}$ phenotype, a greater number of patients with I-phenotype tumors exhibited wall invasion deeper than the submucosal layer (46.0 vs 86.2\%, respectively, $\mathrm{P}<0.01$ ) and high TNM stage (III+IV; 14.3 vs $44.8 \%$, respectively, $\mathrm{P}<0.01)$. Significantly more GI- than G-phenotype tumors were in the larger size category (diameter $>5.0 \mathrm{~cm} ; 54.9 \mathrm{vs} 46.0 \%$, respectively, $\mathrm{P}<0.01$ ). Phenotypic classification did not differ according to patient sex, tumor diameter, or vascular invasion.

\section{K-ras gene mutation and its relationship with clinicopathologic findings}

Our results concerning K-ras mutations are summarized in Table 3, Table 4, and Table 5. K-ras aberrations, all of which were present in codon 12, were detected in $20(12.27 \%)$ of the 163 specimens. Of these, $40 \%(8 / 20)$ carried the mutation GGT $\rightarrow$ GTT, 50\% (10/20) GGT $\rightarrow$ GAT, 5\% $(1 / 20)$ GGT $\rightarrow$ AGT, and 5\% (1/20) GGT $\rightarrow$ TGT (Figure 2). No sequence variations in codons 13 or 61 were detected. Moreover, no significant relationships were noted between clinicopathologic findings and K-ras mutation.

\section{Relationship between phenotypic markers, phenotypic classification, and K-ras mutation}

Relationships between the expression of phenotypic markers and K-ras mutation are shown in Table 6. The frequency of K-ras mutation was 6.3 (4/63), 9.9 (7/71), and 31.0\% 
Table 3. Associations between phenotypic classification, K-ras mutation [N (\%)], and clinicopathologic features.

\begin{tabular}{|c|c|c|c|c|c|c|}
\hline \multirow[t]{2}{*}{ Factor } & \multirow[t]{2}{*}{ Cases } & \multicolumn{3}{|c|}{ Phenotypic classification } & \multicolumn{2}{|c|}{ K-ras mutation } \\
\hline & & $\begin{array}{c}\mathrm{G} \\
(\mathrm{N}=63)\end{array}$ & $\begin{array}{c}\text { GI } \\
(\mathrm{N}=71)\end{array}$ & $\begin{array}{c}\mathrm{I} \\
(\mathrm{N}=29)\end{array}$ & $\begin{array}{l}\text { Positive } \\
(\mathrm{N}=20)\end{array}$ & $\mathrm{P}$ \\
\hline \multicolumn{7}{|l|}{ Sex } \\
\hline Female & 95 & $36(57.1)$ & $45(63.4)$ & $14(48.3)$ & $12(12.6)$ & $>0.05$ \\
\hline Male & 68 & $27(42.9)$ & $26(36.6)$ & $15(51.7)$ & $8(11.7)$ & \\
\hline \multicolumn{7}{|c|}{ Age (years) } \\
\hline$\leq 50$ & 62 & $16(25.4)$ & $34(47.9)$ & $12(41.4)$ & $7(11.3)$ & $>0.05$ \\
\hline$>50$ & 101 & $47(74.6)$ & $37(52.1)$ & $17(58.6)$ & $13(12.9)$ & \\
\hline \multicolumn{7}{|c|}{ Tumor diameter } \\
\hline$\leq 5.0 \mathrm{~cm}$ & 81 & $34(54.0)$ & $32(45.1)^{\mathrm{a}}$ & $15(51.7)$ & $7(8.6)$ & $>0.05$ \\
\hline$>5.0 \mathrm{~cm}$ & 82 & $29(46.0)$ & $39(54.9)^{\mathrm{a}}$ & $14(48.3)$ & $13(15.9)$ & \\
\hline \multicolumn{7}{|c|}{ Depth of wall invasion } \\
\hline $\mathrm{T} 1$ & 57 & $34(54.0)^{\mathrm{b}}$ & $19(26.8)$ & $4(13.8)$ & $5(8.8)$ & $>0.05$ \\
\hline $\mathrm{T} 2$ & 34 & $4(6.3)^{b}$ & $19(26.8)$ & $11(37.9)$ & $3(8.8)$ & \\
\hline T3 & 54 & $18(28.6)^{\mathrm{b}}$ & $25(35.2)$ & $11(37.9)$ & $8(14.8)$ & \\
\hline $\mathrm{T} 4$ & 18 & $7(11.1)^{b}$ & $8(11.3)$ & $3(10.3)$ & $4(22.2)$ & \\
\hline \multicolumn{7}{|c|}{ Vascular invasion } \\
\hline Positive & 32 & $11(17.5)$ & $16(22.5)$ & $5(17.2)$ & $3(9.4)$ & $>0.05$ \\
\hline Negative & 131 & $52(82.5)$ & $55(77.5)$ & $24(82.8)$ & $17(12.9)$ & \\
\hline \multicolumn{7}{|c|}{ Neural invasion } \\
\hline Positive & 52 & $18(28.6)$ & $24(33.8)$ & $10(34.5)$ & $7(13.7)$ & $>0.05$ \\
\hline Negative & 111 & $45(71.4)$ & $47(66.2)$ & $19(65.5)$ & $13(11.7)$ & \\
\hline \multicolumn{7}{|c|}{ Lymph node metastasis } \\
\hline Positive & 69 & $22(34.9)^{\mathrm{c}}$ & $29(40.8)$ & $18(62.1)$ & $7(10.1)$ & $>0.05$ \\
\hline Negative & 94 & $41(65.1)^{\mathrm{c}}$ & $42(59.2)$ & $11(37.9)$ & $13(13.8)$ & \\
\hline \multicolumn{7}{|c|}{ TNM stage } \\
\hline $\mathrm{I}+\mathrm{II}$ & 94 & $54(85.7)^{\mathrm{d}}$ & $24(33.8)$ & $16(55.2)$ & $10(10.6)$ & $>0.05$ \\
\hline III+IV & 69 & $9(14.3)^{d}$ & $47(66.2)$ & $13(44.8)$ & $10(14.5)$ & \\
\hline
\end{tabular}

$\mathrm{G}=$ gastric; $\mathrm{GI}=$ gastrointestinal; $\mathrm{I}=$ intestinal; $\mathrm{TNM}=$ tumor-node-metastasis. ${ }^{\text {a }} \mathrm{P}<0.01$ vs GI phenotype; ${ }^{\mathrm{b}, \mathrm{c}, \mathrm{d}} \mathrm{P}<$ 0.01 vs I phenotype. ${ }^{\mathrm{b}} \mathrm{T} 1$ vs $\mathrm{T} 2+\mathrm{T} 3+\mathrm{T} 4$.

Table 4. Gastric signet ring cell carcinoma cases for which K-ras mutations were identified.

\begin{tabular}{|c|c|c|c|c|c|c|c|c|c|c|c|}
\hline Case No. & $\begin{array}{c}\text { Age } \\
\text { (years)/sex }\end{array}$ & Tumor size & $\begin{array}{c}\text { Invasion } \\
\text { depth }\end{array}$ & $\begin{array}{l}\text { Vascular } \\
\text { invasion }\end{array}$ & $\begin{array}{c}\text { Neural } \\
\text { invasion }\end{array}$ & $\begin{array}{l}\text { Lymph node } \\
\text { metastasis }\end{array}$ & MUC2 & CDX2 & Phenotype & Codon & Mutation \\
\hline 1 & $47 / \mathrm{M}$ & $1 \times 1$ & T1 & - & + & - & + & - & I & 12 & GGT $\rightarrow$ GTT \\
\hline 2 & $72 / \mathrm{F}$ & $3 \times 2.5$ & T1 & - & - & - & - & - & G & 12 & GGT $\rightarrow$ GAT \\
\hline 3 & $42 / \mathrm{M}$ & $1.5 \times 1.5$ & T1 & - & - & + & + & - & GI & 12 & GGT $\rightarrow$ GTT \\
\hline 4 & $45 / \mathrm{F}$ & $6 \times 4$ & T1 & - & - & + & + & + & I & 12 & GGT $\rightarrow$ GTT \\
\hline 5 & $37 / \mathrm{M}$ & $3.5 \times 3$ & T1 & - & - & - & - & + & GI & 12 & GGT $\rightarrow$ GTT \\
\hline 6 & $54 / \mathrm{M}$ & $2 \times 1$ & $\mathrm{~T} 2$ & - & - & - & + & + & I & 12 & GGT $\rightarrow$ AGT \\
\hline 7 & $61 / \mathrm{M}$ & $1 \times 1$ & $\mathrm{~T} 2$ & - & - & - & - & + & GI & 12 & $\mathrm{GGT} \rightarrow \mathrm{TGT}$ \\
\hline 8 & $63 / \mathrm{F}$ & $3 \times 2$ & $\mathrm{~T} 2$ & - & - & - & + & - & I & 12 & GGT $\rightarrow$ GAT \\
\hline 9 & $60 / \mathrm{F}$ & $3 \times 2.5$ & T2 & - & - & - & + & - & GI & 12 & GGT $\rightarrow$ GAT \\
\hline 10 & $77 / \mathrm{M}$ & $\mathrm{x} 4.5$ & $\mathrm{~T} 2$ & + & + & - & + & + & GI & 12 & GGT $\rightarrow$ GAT \\
\hline 11 & $21 / \mathrm{F}$ & $2 \times 1$ & $\mathrm{~T} 2$ & - & - & - & + & + & I & 12 & $\mathrm{GGT} \rightarrow \mathrm{GAT}$ \\
\hline 12 & $52 / \mathrm{M}$ & $3.5 \times 3$ & $\mathrm{~T} 2$ & - & + & - & - & - & G & 12 & $\mathrm{GGT} \rightarrow \mathrm{GTT}$ \\
\hline 13 & $81 / \mathrm{M}$ & $8 \times 6$ & T3 & - & - & - & - & - & G & 12 & GGT $\rightarrow$ GTT \\
\hline 14 & $56 / \mathrm{M}$ & $5 \times 4$ & T3 & - & + & + & + & + & I & 12 & GGT $\rightarrow$ GAT \\
\hline 15 & $71 / \mathrm{M}$ & $2 \times 1$ & T3 & - & + & - & + & + & I & 12 & GGT $\rightarrow$ GTT \\
\hline 16 & $51 / \mathrm{M}$ & $1.5 \times 1.5$ & T3 & + & + & - & - & + & GI & 12 & $\mathrm{GGT} \rightarrow \mathrm{GTT}$ \\
\hline 17 & $48 / \mathrm{M}$ & $3 \times 2$ & $\mathrm{~T} 4$ & - & - & - & + & + & GI & 12 & $\mathrm{GGT} \rightarrow \mathrm{GAT}$ \\
\hline 18 & $78 / \mathrm{M}$ & $3 \times 3$ & $\mathrm{~T} 4$ & + & + & + & - & - & G & 12 & GGT $\rightarrow$ GAT \\
\hline 19 & $76 / \mathrm{F}$ & $1.5 \times 1.5$ & T4 & - & - & + & + & + & I & 12 & GGT $\rightarrow$ GAT \\
\hline 20 & $54 / \mathrm{F}$ & $7.5 \times 7$ & T4 & - & - & + & + & - & I & 12 & $\mathrm{GGT} \rightarrow \mathrm{GAT}$ \\
\hline
\end{tabular}

$\mathrm{M}=$ male; $\mathrm{F}$ = female; $\mathrm{I}=$ intestinal; $\mathrm{G}=$ gastric; $\mathrm{GI}$ = gastrointestinal.

Table 5. K-ras mutations identified among the signet ring cell carcinoma cases in this study.

\begin{tabular}{l|c|c|c|c}
\hline Codon & Mutation & Amino acid change & Cases & $\%$ \\
\hline 12 & GGT $\rightarrow$ GTT & Gly $\rightarrow$ Val & 8 & $40(8 / 20)$ \\
\hline 12 & GGT $\rightarrow$ GAT & Gly $\rightarrow$ Asp & 10 & $50(10 / 20)$ \\
\hline 12 & GGT $\rightarrow$ AGT & Gly $\rightarrow$ Ser & 1 & $5(1 / 20)$ \\
\hline 12 & GGT $\rightarrow$ TGT & Gly $\rightarrow$ Sys & 1 & $5(1 / 20)$ \\
\hline
\end{tabular}

Genetics and Molecular Research 16 (2): gmr16029181 

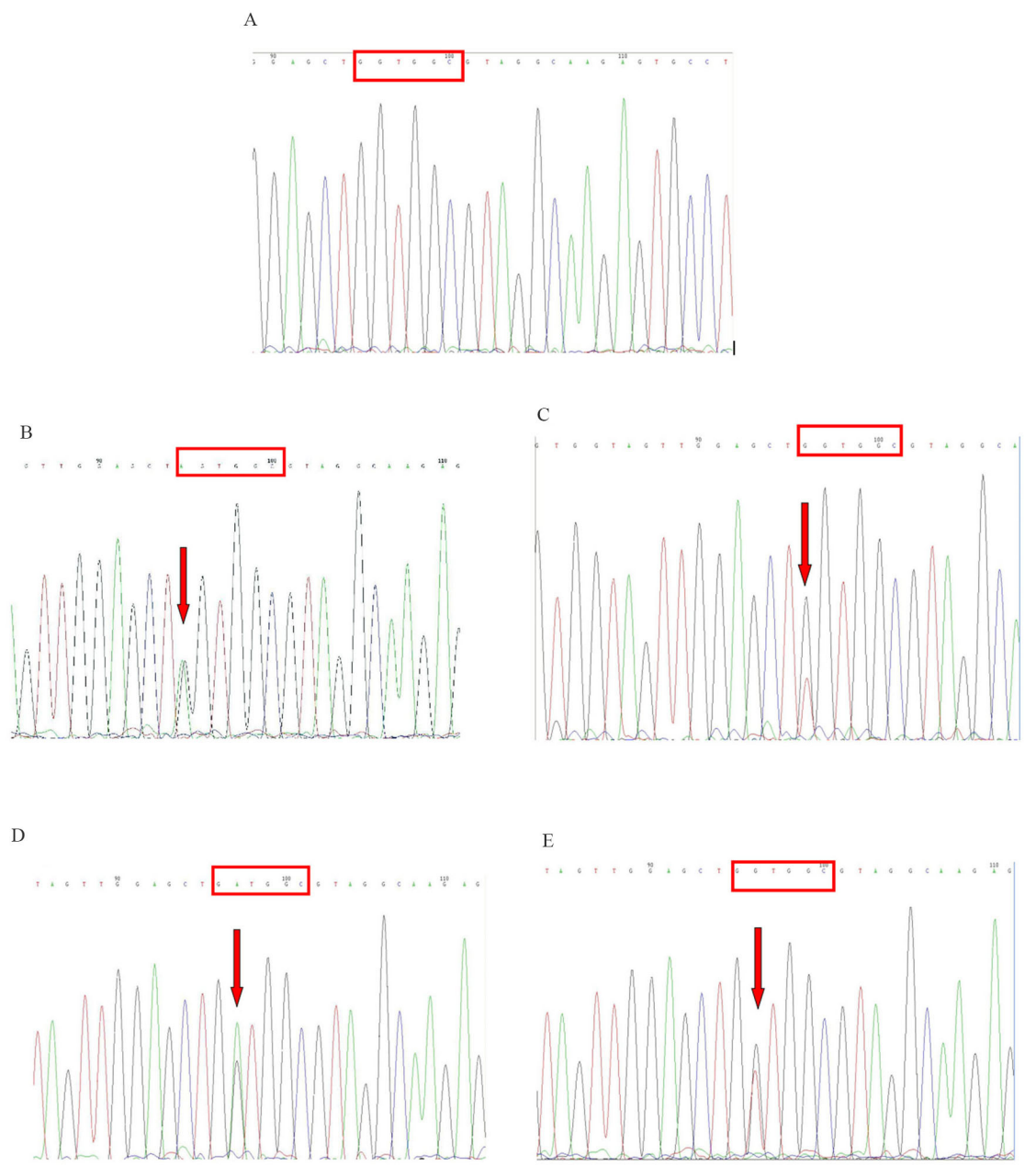

Figure 2. DNA sequencing analysis revealed mutations in codon 12 of the K-ras gene. A. Wildtype sequence of codons 12 and 13 (GGTGGC) on the sense strand; B. and C. GGT $\rightarrow$ AGT (B) and GGT $\rightarrow$ TGT (C) mutations of the first base of codon 12 on the sense strand; D. and E. GGT $\rightarrow$ GAT (D) and GGT $\rightarrow$ GTT (E) mutations of the second base of codon 12 on the sense strand.

(9/29) among gastric SRC carcinomas of the G, GI, and I phenotype, respectively. Considering all 163 cases, the frequency of K-ras mutation was significantly lower among MUC5ACpositive tumors than MUC5AC-negative malignancies ( 8.3 vs $23.3 \%$, respectively, $\mathrm{P}=0.01)$. Similarly, tumors positive for MUC6 demonstrated a significantly lower K-ras mutation rate than those negative for this marker $(2.1 v s 16.4 \%$, respectively, $\mathrm{P}=0.012)$. No significant differences in the incidence of K-ras mutation were observed in relation to MUC1, MUC2, or CDX2 expression. With respect to the relationship between phenotypic classification and K-ras aberration, mutations were observed significantly more often in tumors of the I phenotype $(31.0 \%)$ than in those of the $\mathrm{G}(6.3 \%, \mathrm{P}=0.002)$ and $\mathrm{GI}(9.9 \%, \mathrm{P}=0.009)$ phenotypes. 
Table 6. Associations between phenotypic marker expression, phenotypic classification, and K-ras mutation [N (\%)].

\begin{tabular}{|c|c|c|c|}
\hline & Cases & K-ras mutation $(\mathrm{N}=20)$ & $\mathrm{P}$ \\
\hline \multicolumn{4}{|l|}{ MUC1 } \\
\hline Positive & 34 & $3(8.8)$ & $>0.05$ \\
\hline Negative & 129 & $17(13.2)$ & \\
\hline \multicolumn{4}{|l|}{ MUC5AC } \\
\hline Positive & 120 & $10(8.3)$ & 0.01 \\
\hline Negative & 43 & $10(23.3)$ & \\
\hline \multicolumn{4}{|l|}{ MUC6 } \\
\hline Positive & 47 & $1(2.1)$ & 0.012 \\
\hline Negative & 116 & $19(16.4)$ & \\
\hline \multicolumn{4}{|l|}{ MUC2 } \\
\hline Positive & 76 & $13(17.1)$ & $>0.05$ \\
\hline Negative & 87 & $7(8.0)$ & \\
\hline \multicolumn{4}{|l|}{ CDX2 } \\
\hline Positive & 64 & $9(14.1)$ & $>0.05$ \\
\hline Negative & 99 & $11(11.1)$ & \\
\hline \multicolumn{4}{|c|}{ Phenotypic classification } \\
\hline $\mathrm{G}^{\mathrm{a}}$ & 63 & $4(6.3)$ & 0.002 \\
\hline $\mathrm{GI}^{\mathrm{b}}$ & 71 & $7(9.9)$ & 0.009 \\
\hline I & 29 & $9(31.0)$ & \\
\hline
\end{tabular}

$\mathrm{G}=$ gastric; $\mathrm{GI}=$ gastrointestinal; $\mathrm{I}=$ intestinal. ${ }^{\mathrm{a}} \mathrm{P}=0.002$ vs $\mathrm{I}$-type; ${ }^{\mathrm{b}} \mathrm{P}=0.009$ vs $\mathrm{I}$-type.

\section{DISCUSSION}

In the present study, 163 gastric SRC carcinomas were classified into $\mathrm{G}(63 ; 38.6 \%)$, GI $(71 ; 43.5 \%)$, and I $(29 ; 17.9 \%)$ phenotypes. Previous investigations have reported the frequency of these tumor phenotypes as 17.7-41.1, 28.6-60.1, and 18.5-46.6\%, respectively (Sasaki et al., 1999; Tajima et al., 2001), consistent with the present results. This indicates that both the $\mathrm{G}$ and I phenotype are frequently observed among gastric SRC carcinomas.

Concerning correlations between clinicopathologic findings and phenotypic markers, our data showed that expression of MUC2 was associated with submucosal invasion and lymph node metastasis, and that of CDX2 was connected to tumor size and depth of invasion. MUC5AC expression was found to be inversely associated with invasion of the submucosa. Combined analysis of gastric and intestinal phenotypic markers showed that tumors expressing only the latter had greater malignant potential in terms of invasion and metastasis compared with tumors of other phenotypes. These findings revealed distinct differences in gastric SRC carcinoma aggressiveness according to phenotypic marker expression. Similar results have been described in several Japanese studies. Yamachika et al. (1997) classified 203 gastric SRC carcinomas into $\mathrm{G}$ and I phenotypes with paradoxical concanavalin A(PCS), galactose oxidaseSchiff (GOS), and sialidase-GOS staining, and immunohistochemistry using PGII, SH-9, and TKH-2 antibodies. Their results showed that the proportion of G-phenotype carcinoma cells decreases with invasion depth. Moreover, to define the phenotypes of 54 gastric SRC carcinomas, Bamba et al. (2001) conducted immunohistochemistry to detect MUC2 and M1 expression, and PCS of class III mucin. They found that the larger the mucosal lesion, the more frequently the I phenotype is observed. Aihara et al. (2004) employed MUC2, M1, and MUC6 staining to classify 69 early gastric SRC carcinomas into G and GI phenotypes, finding that the latter correlates with depth of wall invasion. Although there are certain minor differences between their results, these studies demonstrate a tumor phenotypic shift during gastric SRC carcinoma progression. Such carcinomas expressing intestinal phenotypic markers exhibit greater aggressiveness.

Genetics and Molecular Research 16 (2): gmr16029181 
In our study, $12.27 \%$ of the gastric SRC carcinomas tested showed mutational activation of K-ras. To the best of our knowledge, this is the first detailed description of the presence of activating point mutations in the K-ras oncogene in this disease. Notably, Liu et al. (2015) reported that the K-ras mutation rate is higher in SRC carcinoma than other gastric cancer types. Gastric malignancies harboring such oncogenic K-ras mutations might be treated with targeted MEK inhibitor therapy. Moreover, our present work revealed significant associations between phenotypic classification and K-ras mutation in gastric SRC carcinoma. K-ras aberrations were significantly more common among tumors of the I phenotype, and their presence was inversely associated with expression of MUC5AC and MUC6. These findings suggest that phenotypic marker expression is closely related to K-ras mutation in the tumorigenic phase of this malignancy. The divergent behavior of tumors with different phenotypic marker expression patterns indicates the effects of distinct genetic alterations. Morohara et al. (2006) reported that chromosomal changes detected by a comparative genomic hybridization technique considerably differ according to phenotypic marker expression patterns of differentiated-type gastric carcinomas. Yamazaki et al. (2006) demonstrated that APC gene mutation is relatively common in tumors of the I phenotype, but rather rare in those of the $\mathrm{G}$ phenotype, although such aberrations are generally considered to be involved in differentiated-, not diffuse-type carcinomas. Shibata et al. (2003) reported the apoptotic/ proliferative index ratio to be significantly lower in G-phenotype tumors than I-phenotype malignancies. Previous molecular genetic studies have shown that gastric tumorigenesis is a multistep process involving the accumulation of genetic alterations (Stadtländer and Waterbor, 1999). Therefore, prior data and our present findings suggest that different genetic pathways associated with phenotypic marker expression patterns might play a role in the tumorigenesis of gastric SRC carcinoma, leading to variations in tumor behavior.

In conclusion, our present investigation showed that phenotypic classification reflected the behavior of gastric SRC carcinoma. Differences in the biological characteristics of tumors with distinct phenotypes might result from genetic dissimilarities during tumorigenesis. The current analysis was limited by its small sample size; therefore, a larger study population will be included in future work to confirm our results.

\section{Conflicts of interest}

The authors declare no conflict of interest.

\section{REFERENCES}

Aihara R, Mochiki E, Kamiyama Y, Kamimura H, et al. (2004). Mucin phenotypic expression in early signet ring cell carcinoma of the stomach: its relationship with the clinicopathologic factors. Dig. Dis. Sci. 49: 417-424. http://dx.doi. org/10.1023/B:DDAS.0000020496.98711.14

Arber N, Han EK, Sgambato A, Piazza GA, et al. (1997). A K-ras oncogene increases resistance to sulindac-induced apoptosis in rat enterocytes. Gastroenterology 113: 1892-1900. http://dx.doi.org/10.1016/S0016-5085(97)70008-8

Bamba M, Sugihara H, Kushima R, Okada K, et al. (2001). Time-dependent expression of intestinal phenotype in signet ring cell carcinomas of the human stomach. Virchows Arch. 438: 49-56. http://dx.doi.org/10.1007/s004280000307

Guillem P, Billeret V, Buisine MP, Flejou JF, et al. (2000). Mucin gene expression and cell differentiation in human normal, premalignant and malignant esophagus. Int. J. Cancer 88: 856-861. http://dx.doi.org/10.1002/10970215(20001215)88:6<856::AID-IJC3>3.0.CO;2-D

Hongyo T, Buzard GS, Palli D, Weghorst CM, et al. (1995). Mutations of the K-ras and 553 genes in gastric adenocarcinomas from a high-incidence region around Florence, Italy. Cancer Res. 55: 2665-2672.

Genetics and Molecular Research 16 (2): gmr16029181 
Humar B, Fukuzawa R, Blair V, Dunbier A, et al. (2007). Destabilized adhesion in the gastric proliferative zone and c-Src kinase activation mark the development of early diffuse gastric cancer. Cancer Res. 67: 2480-2489. http://dx.doi. org/10.1158/0008-5472.CAN-06-3021

Jung CK, Song KY, Park G, Park CH, et al. (2007). Mucin phenotype and CDX2 expression as prognostic factors in gastric carcinomas. Korean J. Pathol. 41: 139-148.

Lauren P (1965). The two histological main types of gastric carcinoma: diffuse and so-called intestinal-type carcinoma. An attempt at a histo-clinical classification. Acta Pathol. Microbiol. Scand. 64: 31-49.

Lee KH, Lee JS, Suh C, Kim SW, et al. (1995). Clinicopathologic significance of the K-ras gene codon 12 point mutation in stomach cancer. An analysis of 140 cases. Cancer 75: 2794-2801. http://dx.doi.org/10.1002/10970142(19950615)75:12<2794::AID-CNCR2820751203>3.0.CO;2-F

Liu B, Wei J, Wu N, Yu L, et al. (2015). P-054 Evaluation of driver mutations involving in RAS-RAF/PI3K-mToR pathway in gastric signet ring cell carcinoma. Ann. Oncol. 26 (Suppl 4): iv15. http://dx.doi.org/10.1093/annonc/mdv233.54

Morohara K, Tajima Y, Nakao K, Nishino N, et al. (2006). Gastric and intestinal phenotypic cell marker expressions in gastric differentiated-type carcinomas: association with E-cadherin expression and chromosomal changes. J. Cancer Res. Clin. Oncol. 132: 363-375. http://dx.doi.org/10.1007/s00432-005-0062-8

Otsuji E, Yamaguchi T, Sawai K and Takahashi T (1998). Characterization of signet ring cell carcinoma of the stomach. J. Surg. Oncol. 67: 216-220. http://dx.doi.org/10.1002/(SICI)1096-9098(199804)67:4<216::AID-JSO2>3.0.CO;2-B

Sasaki I, Yao T, Nawata H and Tsuneyoshi M (1999). Minute gastric carcinoma of differentiated type with special reference to the significance of intestinal metaplasia, proliferative zone, and p53 protein during tumor development. Cancer 85 : 1719-1729. http://dx.doi.org/10.1002/(SICI) 1097-0142(19990415)85:8<1719::AID-CNCR11>3.0.CO;2-V

Senapati S, Sharma P, Bafna S, Roy HK, et al. (2008). The MUC gene family: their role in the diagnosis and prognosis of gastric cancer. Histol. Histopathol. 23: 1541-1552.

Shibata N, Watari J, Fujiya M, Tanno S, et al. (2003). Cell kinetics and genetic instabilities in differentiated type early gastric cancers with different mucin phenotype. Hum. Pathol. 34: 32-40. http://dx.doi.org/10.1053/hupa.2003.2

Stadtländer CT and Waterbor JW (1999). Molecular epidemiology, pathogenesis and prevention of gastric cancer. Carcinogenesis 20: 2195-2208. http://dx.doi.org/10.1093/carcin/20.12.2195

Tahara E, Semba S and Tahara H (1996). Molecular biological observations in gastric cancer. Semin. Oncol. 23: 307-315.

Tajima Y, Shimoda T, Nakanishi Y, Yokoyama N, et al. (2001). Gastric and intestinal phenotypic marker expression in gastric carcinomas and its prognostic significance: immunohistochemical analysis of 136 lesions. Oncology 61: 212220. http://dx.doi.org/10.1159/000055377

Tajima Y, Yamazaki K, Nishino N, Morohara K, et al. (2004). Gastric and intestinal phenotypic marker expression in gastric carcinomas and recurrence pattern after surgery-immunohistochemical analysis of 213 lesions. Br. J. Cancer 91: 1342-1348. http://dx.doi.org/10.1038/sj.bjc.6602147

Tajima Y, Yamazaki K, Makino R, Nishino N, et al. (2006). Gastric and intestinal phenotypic marker expression in early differentiated-type tumors of the stomach: clinicopathologic significance and genetic background. Clin. Cancer Res. 12: 6469-6479. http://dx.doi.org/10.1158/1078-0432.CCR-06-1339

Yamachika T, Inada K, Fujimitsu Y, Nakamura S, et al. (1997). Intestinalization of gastric signet ring cell carcinomas with progression. Virchows Arch. 431: 103-110. http://dx.doi.org/10.1007/s004280050075

Yamazaki K, Tajima Y, Makino R, Nishino N, et al. (2006). Tumor differentiation phenotype in gastric differentiated-type tumors and its relation to tumor invasion and genetic alterations. World J. Gastroenterol. 12: 3803-3809. http:// dx.doi.org/10.3748/wjg.v12.i24.3803

Yokota T, Kunii Y, Teshima S, Yamada Y, et al. (1998). Signet ring cell carcinoma of the stomach: a clinicopathological comparison with the other histological types. Tohoku J. Exp. Med. 186: 121-130. http://dx.doi.org/10.1620/ tjem.186.121

Genetics and Molecular Research 16 (2): gmr16029181 